\title{
Grasping the Intentions of Others: The Perceived Intentionality of an Action Influences Activity in the Superior Temporal Sulcus during Social Perception
}

\author{
Kevin A. Pelphrey ${ }^{1,2}$, James P. Morris ${ }^{1}$, and Gregory McCarthy ${ }^{1,3}$
}

\begin{abstract}
An explication of the neural substrates for social perception is an important component in the emerging field of social cognitive neuroscience and is relevant to the field of cognitive neuroscience as a whole. Prior studies from our laboratory have demonstrated that passive viewing of biological motion (Pelphrey, Mitchell, et al., 2003; Puce et al., 1998) activates the posterior superior temporal sulcus (STS) region. Furthermore, recent evidence has shown that the perceived context of observed gaze shifts (Pelphrey, Singerman, et al., 2003; Pelphrey et al., 2004) modulates STS activity. Here, using event-related functional magnetic resonance imaging at $4 \mathrm{~T}$, we investigated brain activity in response to passive viewing of goal- and nongoal-directed reaching-to-grasp movements. Participants viewed an animated character making reaching-to-grasp movements either toward (correct) or away (incorrect) from a blinking dial. Both conditions evoked significant posterior STS
\end{abstract}

\section{INTRODUCTION}

The term social cognition refers to our abilities to recognize, manipulate, and behave with respect to socially relevant information, including the ability to construct representations of relations between self and others and to use those representations flexibly to guide our own actions and to predict and interpret others' actions (Adolphs, 2003). Different disciplines emphasize different aspects of social cognition. For example, in social psychology, social cognition describes a range of phenomena including reasoning, stereotyping, and related topics (Kunda, 1999). In neurobiology, Brothers (1990) defines social cognition more narrowly "as the human ability to perceive the intentions and dispositions of others." Definitions of social cognition commonly link it to social behavior and include concepts such as social perception, theory of mind (the ability to make inferences about the mental

\footnotetext{
${ }^{1}$ Brain Imaging and Analysis Center, Duke University, ${ }^{2}$ University of North Carolina, ${ }^{3}$ Department of Veterans Affairs Medical Center, Durham, North Carolina
}

activity that was strongly right lateralized. By examining the time course of the blood oxygenation level-dependent response from areas of activation, we observed a functional dissociation. Incorrect trials evoked significantly greater activity in the STS than did correct trials, while an area posterior and inferior to the STS (likely corresponding to the MT/V5 complex) responded equally to correct and incorrect movements. Parietal cortical regions, including the superior parietal lobule and the anterior intraparietal sulcus, also responded equally to correct and incorrect movements, but showed evidence for differential responding based on the hand and arm (left or right) of the animated character used to make the reaching-to-grasp movement. The results of this study further suggest that a region of the right posterior STS is involved in analyzing the intentions of other people's actions and that activity in this region is sensitive to the context of observed biological motions. states of others), and attributional style (the way one tends to explain others' behavior).

Cognitive neuroscientists are beginning to elucidate the neural structures and circuits involved in human social cognition and the social brain. Work within our own laboratory has focused on the role of the superior temporal sulcus (STS) region in social perception (Pelphrey, Mitchell, et al., 2003; Pelphrey, Singerman, Allison, \& McCarthy, 2003; Pelphrey, Viola, \& McCarthy, 2004; Puce, Allison, Bentin, Gore, \& McCarthy, 1998). As defined by Allison, Puce, and McCarthy (2000), social perception refers to the initial stages of evaluating the social communicative intentions of others by analysis of eye-gaze direction, facial expressions, body movements, and other types of biological motion. An explication of the neural substrates for social perception is an important component in the emerging field of social cognitive neuroscience.

Contemporary thought suggests that the human posterior STS region serves as a critical interface between the dorsal and ventral visual streams, thus, supporting the integration of object and spatial information (Karnath, 2001; Perrett et al., 1989). The functional organization of 
the STS region has been studied extensively in both human and nonhuman primates. Employing single-cell recordings, Perrett and his colleagues identified cells in the macaque STS that selectively respond to cues from head and gaze direction (Perrett et al., 1989; Perrett, Smith, Potter, et al., 1985) as well as different kinds of biological motion including observation of goal-directed reaching-to-grasp action sequences (Perrett et al., 1989; Perrett, Smith, Mistlin, et al., 1985). Functional neuroimaging work in humans has identified a region in the posterior STS, which is thought to be the human homologue for monkey STS (reviewed by Karnath, 2001).

It is now generally accepted that the human posterior STS is involved in processing viewed biological motion (e.g., Beauchamp, Lee, Haxby, \& Martin, 2003; Pelphrey, Mitchell, et al., 2003; Grossman \& Blake, 2001, 2002; Vaina, Solomon, Chowdhury, Sinha, \& Belliveau, 2001; Grèzes et al., 2001; for reviews, see Allison et al., 2000; Decety \& Grèzes, 1999; Bonda, Petrides, Ostry, \& Evans, 1996). This region is functionally and anatomically distinct from the more posterior and inferior region MT or V5 complex (MT/V5), which is more generally sensitive to both nonbiological and biological motion (McCarthy et al., 1995; Tootell et al., 1995; Watson et al., 1993; Zeki, 1991). In addition to its role in the perception of biological motion, there is more recent evidence that similar to the STS in the macaque, the posterior STS in humans is involved in the analysis and interpretation of the intentions of others' goal-directed movements and actions (e.g., Blakemore et al., 2003; Pelphrey, Singerman, et al., 2003; Pelphrey et al., 2004; for a review, see Allison et al., 2000).

In an early functional magnetic resonance imaging (fMRI) study, our laboratory first demonstrated the role of the STS in eye-gaze processing (Puce et al., 1998). Subsequently, we investigated the degree to which eyegaze-evoked activity in the STS is modulated by the context of the perceived eye movement, that is, when the gaze shift correctly or incorrectly acquires a visual target. In that study, a strong effect of context was observed in the right posterior STS in which observation of gaze shifts away from the target evoked a hemodynamic response (HDR) with extended duration and greater amplitude compared to gaze shifts toward the target (see Pelphrey, Singerman, et al., 2003: Figure 6). Thus, we made the novel observation that the perceived context or intentionality of a specific biological motion influences activity in the human STS.

Since then, we have examined whether the STS participates in the visual analysis of social information conveyed by gaze shifts in a more overtly social encounter-a stranger walking toward and past the subject in a virtual hallway. Subjects viewed an animated figure that walked toward them and shifted his neutral gaze either toward (mutual gaze) or away (averted gaze) from them. We found that mutual gaze evoked greater activity in the STS compared to averted gaze (Pelphrey et al., 2004,
Figure 3). We reasoned that if eye-gaze-related STS activity reflects the operation of a simple eye-movement detector (or more generally, a biological motion detector), the region should not respond differentially to mutual and averted gaze or to target- versus non-targetdirected movements.

In summary, our work to date suggests that activity in the STS evoked during observation and interpretation of others' eye movements is exquisitely sensitive to the context within which those actions are embedded (i.e., approach vs. avoidance or goal- vs. non-goal-directed). It is not yet clear whether this sensitivity extends beyond eye gaze to other categories of biological motion such as walking, reaching, or grasping movements.

Here, using event-related fMRI at $4 \mathrm{~T}$, we investigated brain activity in response to passive viewing of reachingto-grasp movements. Participants viewed an animated male character making reaching movements either toward (correct) or away (incorrect) from a blinking dial. To ensure that activation differences were not simply due to motion per se, incorrect and correct trials contained the same amount of motion. Based on prior findings from our laboratory (Pelphrey et al., 2004; Pelphrey, Mitchell, et al., 2003), we hypothesized that the STS region would differentiate correct and incorrect trials while other motion-sensitive regions (e.g., the MT/ V5 complex, parietal cortex) would respond equally to our two conditions.

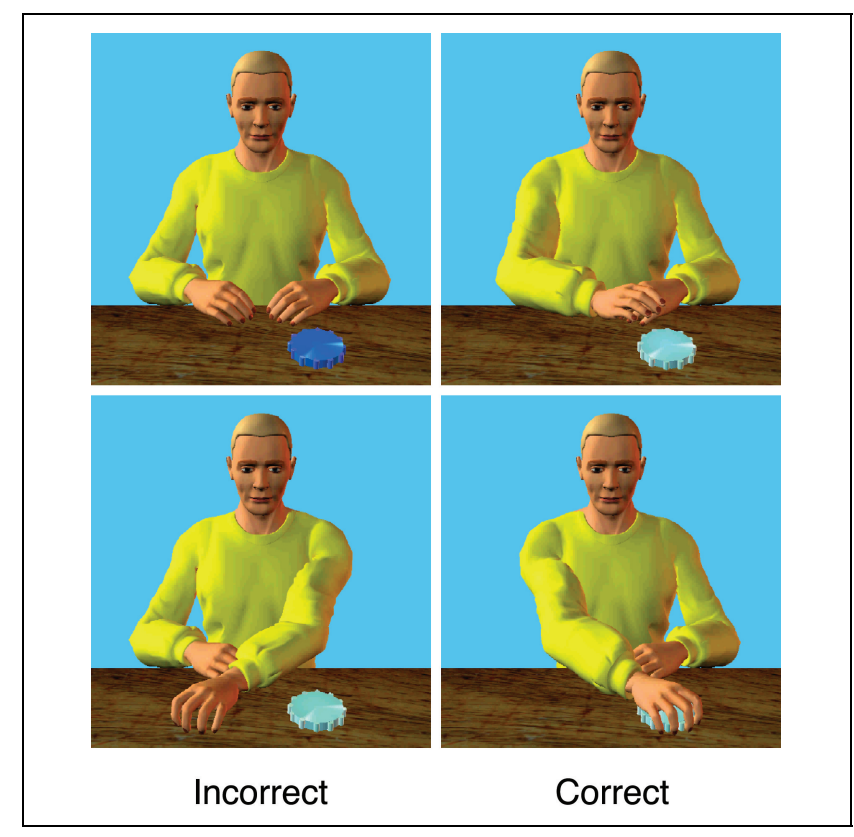

Figure 1. Experimental conditions. Both conditions began with the dial flashing between light and dark blue for $1 \mathrm{sec}$. During correct trials (right panels), the animated figure made a reaching-to-grasp movement toward the blinking dial. During incorrect trials (left panels), the animated character made the same reaching-to-grasp movement to empty space. 


\section{RESULTS}

The reaching-to-grasp sequence (Figure 1) strongly activated eight regions of interest (ROI) comprising four anatomical locations and two hemispheres. Statistics for these ROI, including peak activity stereotaxic coordinates, laterality indices, and voxel counts, are listed in Table 1. As illustrated in Figure 2, bilateral activity was localized to the posterior STS (panels 1, 3, 4, 6, and 9), the occipito-temporal sulcus/fusiform gyrus (OTS, panels 4 and 9-10), the parieto-temporo-occipital fossa (PTOF, panels 1-2 and 6-8), and the anterior intraparietal sulcus (AIPS, panels 4-5 and 9).

Within the posterior STS, the average peak amplitude in the right hemisphere $(M=0.42 \% ; S E=0.08 \%)$ was greater than that in the left hemisphere $(M=0.27 \%$; $S E=0.03 \%)$ regardless of experimental condition, $F(1,11)=5.88, p=.034$. This finding, combined with an observed laterality index (LI) of 2.41 for the STS (Table 1), confirms previous reports of strong right hemisphere laterality for processing biological motion in the STS (Pelphrey et al., 2004; Pelphrey, Mitchell, et al., 2003; Pelphrey, Singerman, 2003; Wright, Pelphrey, Allison, McKeown, \& McCarthy, 2003; Puce et al., 1998).

The location of the right posterior STS activity observed in this study (Figure 2, panel 6, peak coordinates: $x=54, y=-47, z=11$ ) is consistent with the area of STS identified by Iacoboni et al. (2001) in their study of observed and/or imitated finger movements (see their Figure 1, peak coordinates: $x=57, y=-50, z=16$ ). This region corresponds to the posterior portion of the main branch of the STS complex and its ascending limb. The current STS activity can also be compared to a

Table 1. Summary of Observed Regions of Activation

\begin{tabular}{lcrrrrr}
\hline Region & Side & \multicolumn{1}{c}{$x$} & \multicolumn{1}{c}{$y$} & $z$ & Nvox & LI \\
\hline STS & $\mathrm{R}$ & 57 & -47 & 11 & 65 & 2.41 \\
& $\mathrm{~L}$ & -47 & -80 & 11 & 27 & \\
\multirow{2}{*}{ OTS } & $\mathrm{R}$ & 43 & -62 & -13 & 21 & 0.53 \\
& $\mathrm{~L}$ & -30 & -63 & -13 & 40 & \\
\multirow{2}{*}{ PTOF } & $\mathrm{R}$ & 60 & -64 & 03 & 19 & 0.58 \\
& $\mathrm{~L}$ & -37 & -84 & 03 & 33 & \\
AIPS & $\mathrm{R}$ & 33 & -53 & 55 & 05 & 0.20 \\
& $\mathrm{~L}$ & -24 & -53 & 58 & 25 & \\
\multirow{2}{*}{ MFG* } & $\mathrm{R}$ & 55 & 11 & 45 & 23 & 3.29 \\
& $\mathrm{~L}$ & -55 & -02 & 49 & 07 & \\
\hline
\end{tabular}

Nvox $=$ number of voxels in the ROI; $x, y$, and $z=$ stereotaxic coordinates of the peak of activation with an ROI; $\mathrm{R}=$ right hemisphere; $\mathrm{L}=$ left hemisphere; $\mathrm{LI}=$ laterality index (calculated as the Nvox R / Nvox L).

*This region was identified at a lower statistical threshold of $p<.001$. Other regions were identified with a threshold of $p<.0001$. previous report by our laboratory of activity evoked by observed mouth (peak coordinates: $x=50, y=-49$, $z=3$ ) and eye (peak coordinates: $x=49, y=-49, z=3$ ) movements (see Puce et al., 1998, Figure 9).

The average time courses from the ROI are given in Figure 3. Notably, in the STS, the two waveforms diverged a few seconds after the onset of the trials, and the blood oxygenation level-dependent (BOLD) signal change was substantially greater for incorrect movements (red line with circles) relative to correct movements (black line with diamonds), $F(1,11)=16.20, p=$ .002 . The Hemisphere $\times$ Condition interaction was not significant, indicating a similar pattern of effects for the left and right STS. The other three ROI did not show differential activity by hemisphere or condition. Indeed, the BOLD percent signal change time courses for correct and incorrect movements in these ROI fully overlapped (Figure 3, panels 2-4), and this pattern did not differ by hemisphere.

The PTOF peak activation coordinates (right: $x=60$, $y=-64, z=3$; left: $x=-37, y=-84, z=3$ ) observed are consistent with those published by our group (McCarthy et al., 1995) and others (Tootell et al., 1995; Watson et al., 1993; Zeki et al., 1991) for the more general and functionally defined motion-sensitive region MT/V5. Moreover, we have consistently observed this region, which is posterior and inferior to the STS and located close to the top of the ascending limb of the inferior temporal sulcus, to be activated by biological and nonbiological motion (Pelphrey et al., 2004; Pelphrey, Mitchell, et al., 2003; Pelphrey, Singerman, et al., 2003; Puce et al., 1998; McCarthy et al., 1995). ${ }^{1}$

Similarly, the AIPS appeared to be localized to a region near the human anterior intraparietal area often reported to be engaged during motor execution of grasping movements of the hand (Culham, 2004; Culham et al., 2003; Culham \& Kanwisher, 2001; Binkofski et al., 1998) and appeared to be close to the parietal cortex activity observed by Buccino et al. (2001) and Iacoboni et al. $(1999,2001)$ in fMRI studies of individuals viewing and executing hand and finger movements. Given these similarities and the finding of "mirror" properties in these parietal regions (e.g., Buccino et al., 2001), we performed an exploratory analysis of this ROI after re-epoching the data with respect to the hand (left or right) used by the animated character to make the reaching-to-grasp movement, while collapsing across correct and incorrect movements (i.e., collapsing across correct and incorrect trials).

An interesting pattern of effects emerged from this exploratory analysis. As shown in Figure 4, activity in the left AIPS was greater for observed movements of the left hand. Activity in the right AIPS was greater for observed movements of the right hand. Supporting this pattern of results, a 2 (hemisphere: right, left) $\times 2$ (hand: right, left) repeated measures analysis of variance (ANOVA) revealed a significant Hemisphere $\times$ Hand interaction, 
Figure 2. Activation maps reflecting the intersection of correct and incorrect trials. The maps show the intersection containing voxels with significant positive response to both correct and incorrect trials. Significant activation was localized to the posterior STS (panels 1, 3, 4, 6, and 9), the OTS (panels 4-5 and 9-10), the PTOF (panels 1-2 and 6-8), and the AIPS region (panels 4-5 and 9).

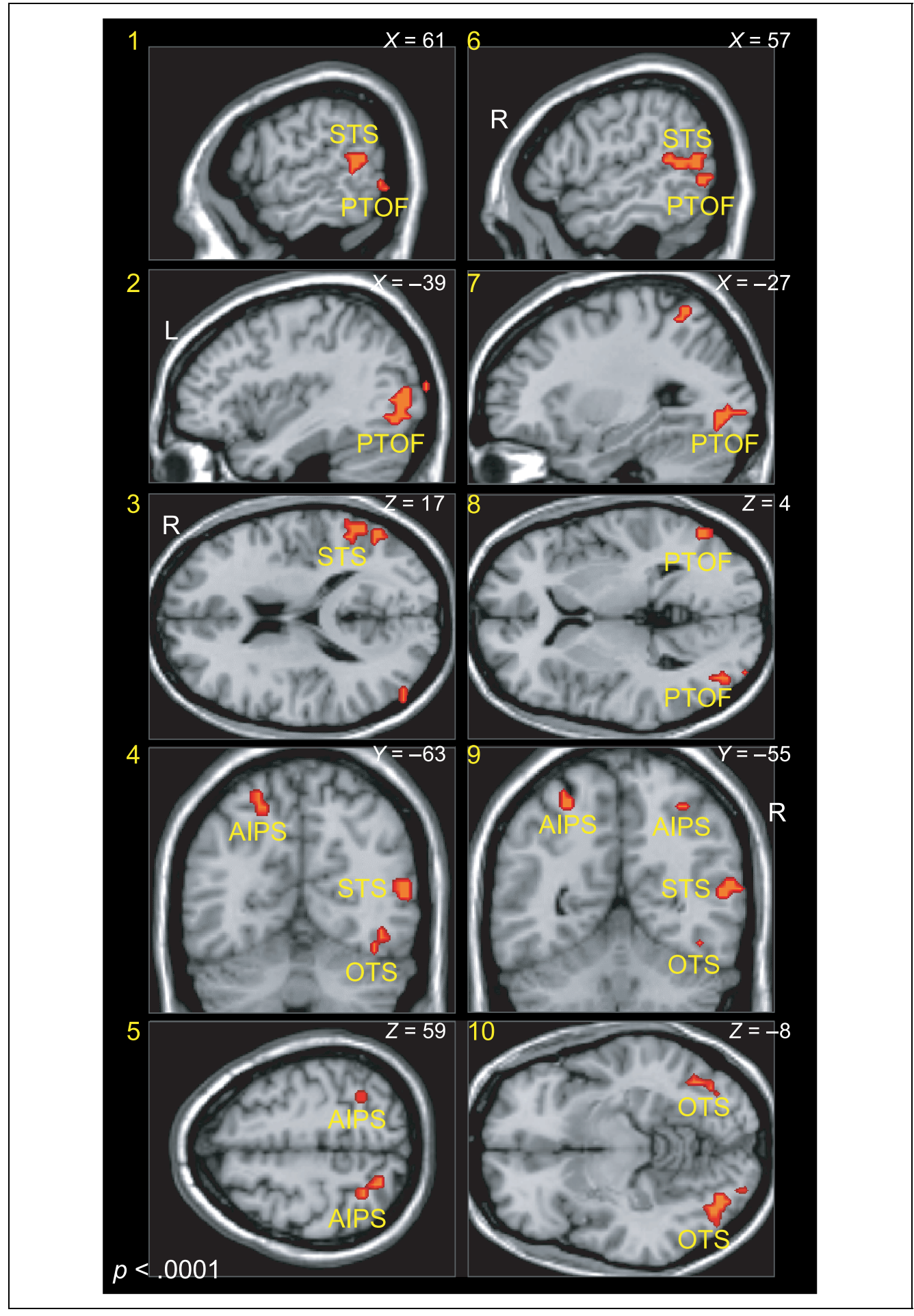

$F(1,11)=9.04, p=.012$. No other ROI, including the STS and MT/V5 complex, exhibited this pattern of effects.

To identify potentially important regions of activation missed in our initial analysis of the data, we lowered our statistical threshold to a voxelwise uncorrected $p<.001$ (two-tailed) and a spatial extent of three contiguous uninterpolated voxels. The top two panels of Figure 5 show a region of activation localized to the posterior middle frontal gyrus (MFG) that emerged from this exploratory analysis. Inspection of the waveforms from this ROI (Figure 5, lower panel) revealed that much like the STS, the MFG responded more strongly to incorrect than to correct reaching-to-grasp movements, $F(1,11)=6.58, p=.028$. The effect of hemisphere and the Hemisphere $\times$ Condition interaction were not significant, although more voxels were 


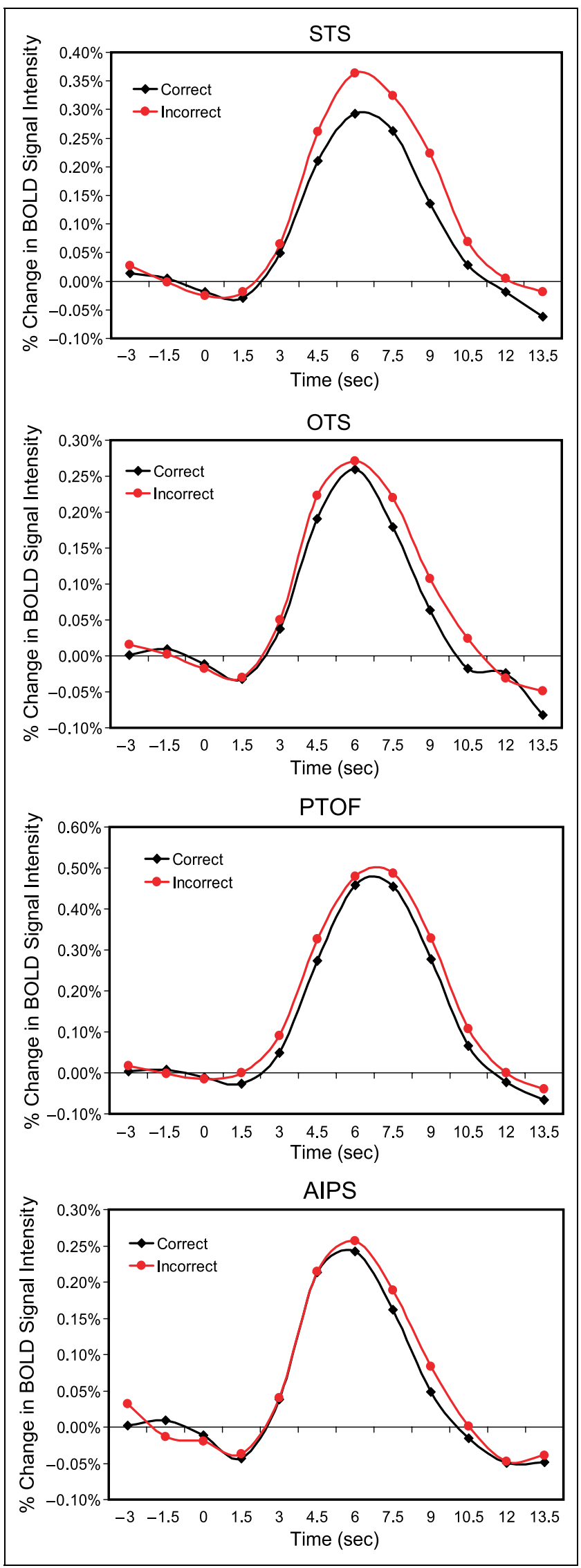

active in the right MFG than in the left as indicated by a laterality index (LI) of 3.29 (Table 1).

\section{DISCUSSION}

Prior work from our laboratory demonstrated a functional dissociation between a specialized region of the STS that differentiates biological from nonbiological motion and a region more posterior and inferior (likely corresponding to the MT/V5 complex), which responds equally to biological and nonbiological motion (Pelphrey, Mitchell, et al., 2003; Puce et al., 1998). We subsequently demonstrated that this STS region is also sensitive to at least two aspects of the context within which the biological motion is perceived: goal- versus non-goal-directed (Pelphrey, Singerman, et al., 2003) and approach versus avoidance (Pelphrey et al., 2004). The current study is unique in that we have extended our initial findings from paradigms featuring dynamic gaze shifts to a paradigm that features the passive viewing of naturalistic reaching-to-grasp movements that are either goal- or non-goal-directed. Our current results, combined with our previous findings in this area, further suggest that the posterior STS is an important component of the neural architecture supporting social perception; allowing for effortless perception, consolidation, and revision of information regarding the intentions, goals, and actions of agents in our environment.

Social perception incorporates what Dennett (1987) described as the human tendency to take an "intentional stance" in predicting and interpreting the behaviors of others:

Here is how it works: first you decide to treat the object whose behavior is to be predicted as a rational agent; then you figure out what beliefs that agent ought to have, given its place in the world and its purpose. Then you figure out what desires it ought to have, on the same considerations, and finally you predict that this rational agent will act to further its goals in the light of its beliefs. A little practical reasoning from the chosen set of beliefs and desires will in many-but not all-instances yield a decision about what the agent ought to do; that is what you predict the agent will do.

(The Intentional Stance, p. 17)

Figure 3. Average BOLD signal change time courses from the activated voxels in the STS, OTS, PTOF, and AIPS regions. The red waveforms with circles as point markers represent the average BOLD signal change evoked by incorrect trials, whereas the black waveforms with diamonds as point markers represent the average BOLD signal change evoked by correct trials. Although the STS showed significant activation differences between correct and incorrect trials, other motion-sensitive areas of the brain, including OTS, PTOF, and AIPS, did not show any significant difference in BOLD signal change for correct and incorrect trials. 


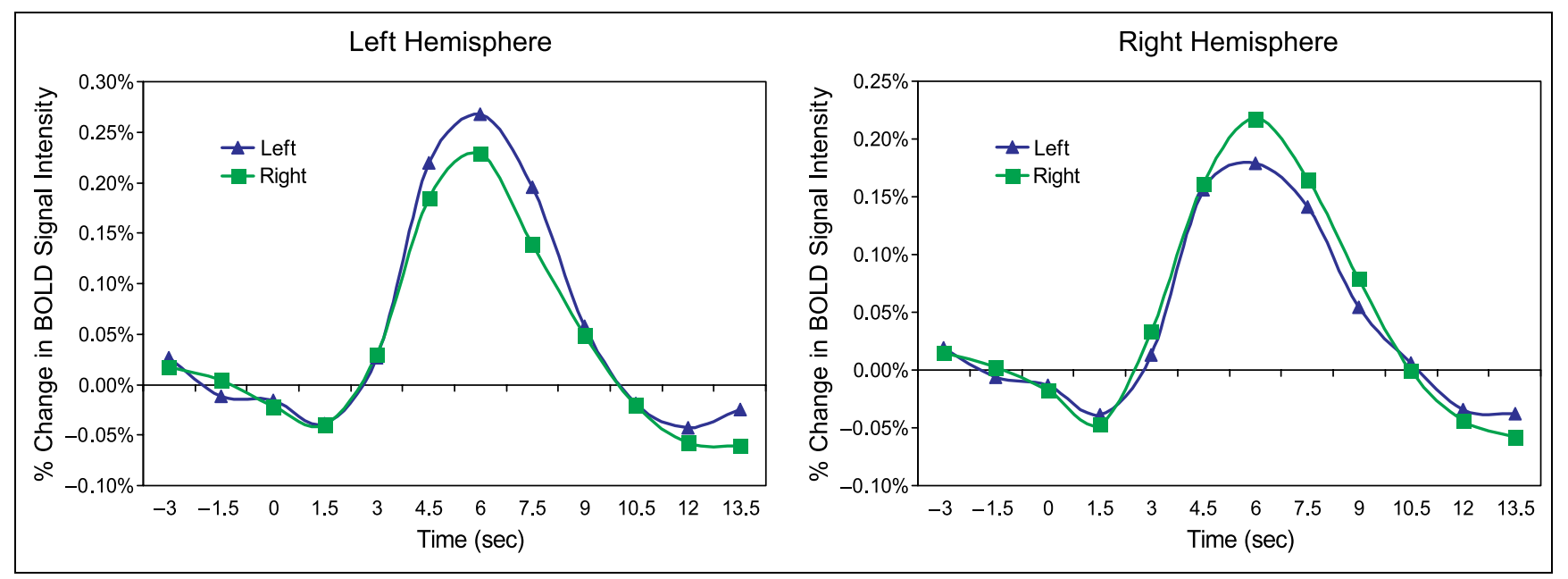

Figure 4. Average BOLD signal change time courses from the activated voxels in the right and left AIPS, in response to left- and right-handed reaching-to-grasp movements. The left AIPS showed greater activation to left hand and arm reaching-to-grasp movements relative to the right hand and arm reaching-to-grasp movements, while the right AIPS showed greater activation to reaching-to-grasp movements performed with the right hand and arm relative to those movements performed with the left hand and arm. This interaction was significant.

The ubiquity (e.g., Heider \& Simmel, 1944), early emergence in development (e.g., Astington \& Gopnik, 1991; Gelman \& Spelke, 1981), and disruption in autism

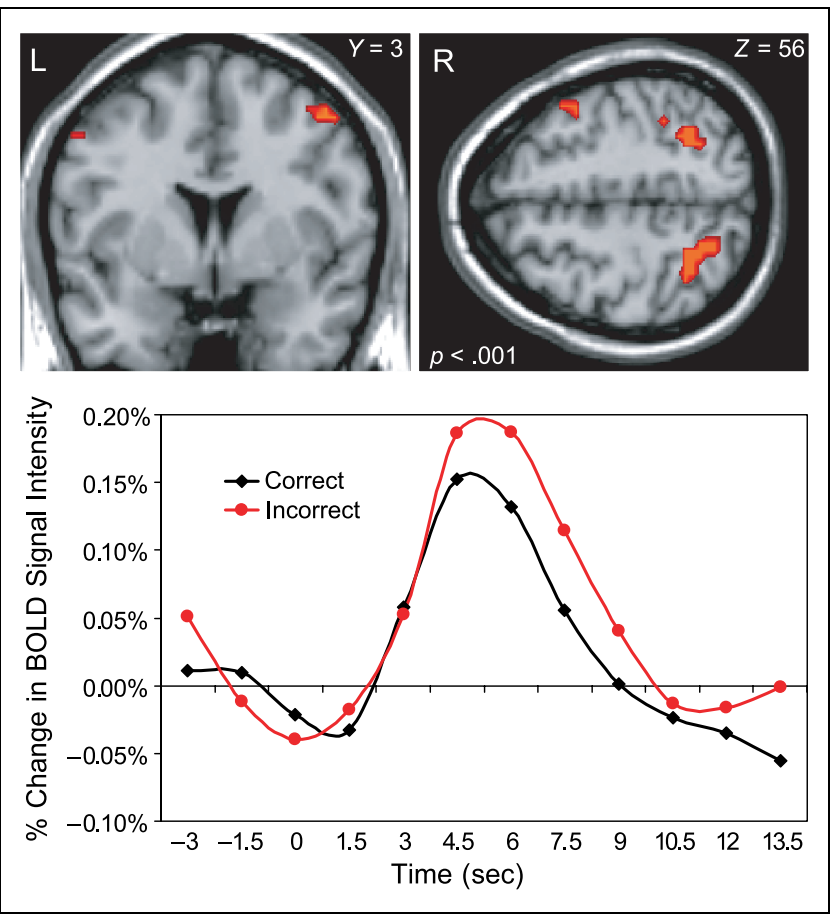

Figure 5. Activation map and average BOLD signal change time courses from the activated voxels in the MFG in response to correct and incorrect movements. The activation map reflects the intersection of correct and incorrect trials (i.e., voxels with a significant response to both correct and incorrect trials). The red waveform with circles as point markers represents the average BOLD signal change evoked by incorrect trials, while the black waveform with diamonds as point markers represents the average BOLD signal change evoked by correct trials (bottom panel). The MFG responded more strongly to incorrect than to correct reaching-to-grasp movements. (e.g., Klin, 2000; Baron-Cohen, 1995) of the human propensity to adopt an intentional stance support the notion that it is a fundamental aspect of human social perception and social cognition.

The term intentionality has traditionally been used by philosophers (e.g., Brentano, Husserl) to refer to a state about or directed toward another state (e.g., the belief that the market is about to rise is a belief about the current state of the New York Stock Exchange). More recently, this term has come to be used more narrowly as a derivative of the verb intend and implies doing something on purpose (Feldman \& Reznick, 1996). Anscombe (1957) used intentional action to describe an act directed at bringing about some state of affairs, and Goschke and Kuhl (1993) used intention to denote that an individual is committed to performing an activity. In this article, we adopt this more "folk psychological" usage and apply the quality intentional to an agent's actions when an observer believes that the actions are based on some degree of awareness and are executed deliberately.

In the current study, we tested the hypothesis that the posterior STS would differentiate between observed goal- (correct) and non-goal-directed (incorrect) action sequences. We found that the passive viewing of nongoal-directed reaching-to-grasp movements evoked significantly stronger activity in the posterior STS than did goal-directed movements. This finding is consistent with a similar finding in the context of target- and non-target-directed gaze shifts (Pelphrey, Singerman, et al., 2003). We interpret this finding to suggest that this region of the STS is sensitive to the intentionality and appropriateness of biological motion. In this interpretation, we propose that the blinking dial serves as a target stimulus capable of eliciting a specific action (reaching-to-grasp the dial) from the animated charac- 
ter. This establishes an expectation for the character's subsequent reaching behavior based on the subject's implicit predictions concerning the rationality of the actor. When the character makes a non-goal-directed action toward empty space, processing demands are increased due to a violation of the subject's expectation. In essence, this violation requires the subject to revise his or her initial expectation and thus demands greater perceptual processing of the action sequence. In Dennett's (1987) framework: A non-goal-directed action sequence violates the subjects' "intentional stance" and therefore their expectations about what the actor "ought to do" given the demands of the action context.

Whereas the theoretical impetus for our current methodology was to test an a priori hypothesis regarding the role of the posterior STS in social perception, the fact that we acquired whole-brain fMRI data afforded us the opportunity to look for interesting differences in other brain regions. Our analyses revealed only one other brain region that differentiated correct and incorrect trials. We found significant activation differences in the MFG, where incorrect trials evoked a significantly larger BOLD response than did correct trials. Activity was greater in the right than in the left MFG for correct and incorrect trials. While these activation differences were not predicted, they are consistent with other fMRI studies that have probed the function of the right MFG. For example, Blakemore et al. (2003) found significant right MFG activation when participants attended to both the contingency and animacy of simple geometric shapes moving on the screen in coordinated motion, but not when subjects attend to contingency or animacy alone. The authors interpreted their results as implicating the right MFG in a neural circuit supporting theory of mind. The current study did not feature manipulations of attention to contingency; but it seems plausible that our stimuli created for the subject a natural contingency between the agent and the target. From this perspective, significant activity in the right MFG is not surprising, given current thought on the role of this region in processes of social perception and social cognition.

The finding of activity in regions of the parietal cortex evoked by observation of an actor's reaching-to-grasp movements is consistent with prior work by Rizzolatti and colleagues concerning neurons (in monkeys) and brain regions (in humans) that respond when the subject both executes an action and when they view an agent performing the same action (Buccino et al., 2001; Binkofski et al., 1999; Iacoboni et al., 1999; Rizzolatti \& Arbib, 1998; see also Grèzes et al., 2002). These "mirror neurons" have been identified in area F5 of the monkey (e.g., Rizzolatti \& Arbib, 1998; Rizzolatti et al., 1996) and "mirror regions" have been localized to premotor, parietal, and posterior temporal areas in humans (e.g., Grèzes et al., 2002; Iacoboni et al., 1999,
2001). Grèzes et al. (2002) have proposed a "mirror circuit" consisting of the intraparietal sulcus, dorsal premotor cortex, bilateral STS, and the right parietal operculum.

Prior studies have not, however, reported differential activity as a function of which hand and arm are observed to make a reaching-to-grasp action. Movements of the actor's left hand and arm evoked significantly greater activity in the left AIPS relative to activity evoked by right hand and arm movements. By contrast, right hand and arm movements evoked significantly greater activity in the right AIPS relative to the activation evoked by left hand and arm movements. Others have suggested that mirror regions of the human brain are involved in imitation (e.g., Arbib et al., 2000; Iacoboni et al., 1999). Our findings appear to support the proposed role of the AIPS in imitation when one considers the results of behavioral research showing that when two individuals face one another and one is asked to imitate the movement of the other, the resultant imitation appears to be a "mirror" image of the imitated action as opposed to imitation of the anatomically correct movement (Avikainen et al., 2003; Bekkering et al., 2000; Wapner \& Cirillo, 1968), and provision of mirror-image-like movements facilitates performance in simple learning tasks (Brass et al., 2000).

The parietal region of activity identified in this study responded equally to correct and incorrect reaching-tograsp movements. This appears to be in contrast to Buccino et al. (2001), who found that the parietal cortex was active in response to viewing videotaped object-related actions (e.g., biting an apple and chewing) but did not respond to the same videotaped actions in the absence of the object (e.g., grasping a ball in the absence of the ball). A critical design difference between that study and ours is that in our study, the object was always in view. So, in spite of the fact that the reaching-to-grasp movement sometimes brought the hand in contact with the dial and sometimes not, the movement was always object-related in the sense that the blinking dial signified the onset of the trial and the subject always saw a movement that conformed to the affordances of the dial's shape, size, and function. Indeed, the differential pattern of incorrect versus correct effects for the STS and the AIPS suggests a functional dissociation for these two regions with regard to their roles in social perception. The STS participates in the visual analysis of others' actions via perceptual analysis of biological motion and sensitivity to the intentionality and appropriateness of an action in a given context. The AIPS is involved in processing biological motion via analysis of an action in relation to objects in the visual field. It remains to be determined whether the separate analyses performed in these regions would look the same for other people in the visual field as compared to objects. 


\section{METHODS}

\section{Subjects}

Twelve healthy young adults ( 7 women and 5 men), ranging in age from 19 to 28 years ( $M=23$ years), participated in this study. All participants were right handed, had normal or corrected-to-normal vision, and were screened for medical, neurological, and psychiatric illnesses and use of psychotropic medications. All subjects gave informed consent prior to participation, as per the guidelines of the Duke University Medical Center Institutional Review Board.

\section{Experimental Design}

Two experimental conditions were created using the Poser 4.0 software program (Curious Labs, Santa Cruz, CA) (Figure 1). In each condition, subjects viewed an animated male character seated at a virtual wooden workbench. Positioned on either side (side varied across runs) of the workbench, out of the character's direct line of sight, was a dark blue dial (Figure 1, upper left panel). To avoid confounding reaching-to-grasp movements with direction of eye gaze, the animated character's gaze was fixated on the center of the table and not on the flashing dial. The dial began to flash rapidly from dark blue to light blue (to convey the impression of a lighted dial requiring action) at the beginning of each trial. After flashing for $1 \mathrm{sec}$, the dial remained light blue and the animated character made a fluid reaching movement toward the dial with his contralateral arm (Figure 1, upper right panel) or away from the dial with his ipsilateral arm (Figure 1, lower left panel). This reach was followed by a grasping and turning movement toward the inside thumb on either hand (Figure 1, lower right panel). Correct trials were defined as those where the figure made movements toward the dial (Figure 1, lower right panel), and incorrect trials were those where the figure made the same movement toward empty space (Figure 1, lower left panel). After making the turning motion, the character immediately moved his arm and hand back to the initial resting position. Overall, the impression was one of a realistic, fluid, and purposeful reaching-to-grasp and twisting motion. We emphasize that the mechanical aspects of the reaching movements were identical across correct and incorrect trials.

Using the program CIGAL (Voyvodic, 1999), stimuli were presented at XGA resolution via LCD goggles. Subjects were instructed only to pay attention to the stimuli at all times. Each vignette lasted $3 \mathrm{sec}$, and trials were separated by a 15-sec intertrial interval, during which the subject viewed the scene with the character's hands at rest (upper left panel of Figure 1). It should be noted that trials appeared to be seamless to the subject in that each trial began with the character's hands at rest and each trial ended in the exact same manner, without any visual cues signaling the next trial. Trials were randomized within 7.75 -min runs containing 24 trials and each subject viewed 8 runs for 192 trials (96 correct, 96 incorrect). In four blocks, the dial was positioned on the left side of workbench relative to the animated character so that correct trials occurred when the character reached with his right hand toward the dial. This pattern was reversed for the other four experimental runs.

\section{Imaging}

Scanning was performed on a General Electric 4-T LX NVi MRI scanner system (General Electric, Milwaukee, WI) equipped with $41 \mathrm{mT} / \mathrm{m}$ gradients and using a quadrature birdcage radio frequency (RF) head coil for transmit and receive. The subject's head was immobilized using a vacuum cushion and tape. Sixty-eight axial images were acquired using a 3-D fast spoiled gradientrecalled pulse sequence $(\mathrm{TR}=500 \mathrm{msec}$; $\mathrm{TE}=20 \mathrm{msec}$; $\mathrm{FOV}=24 \mathrm{~cm}$; image matrix $=256^{2}$; voxel size $=0.9375 \times$ $0.9375 \times 1.9 \mathrm{~mm}$ ) and used for coregistration with the functional data. Functional images were acquired using a gradient-recalled inward spiral pulse sequence (Guo \& Song, 2003; Glover \& Law, 2001) sensitive to BOLD contrast $(\mathrm{TR}=1500 \mathrm{msec} ; \mathrm{TE}=35 \mathrm{msec} ; \mathrm{FOV}=24 \mathrm{~cm}$; image matrix, $64^{2} ; \alpha=62^{\circ}$; voxel size $=3.75 \times 3.75 \times$ $3.8 \mathrm{~mm}$; 34 axial slices) that allowed whole-brain coverage. The anterior and posterior commissures were identified in the midsagittal slice and used as landmarks for the prescription of BOLD contrast images. A semiautomated high-order shimming program ensured global field homogeneity. Runs consisted of 310 time points and began with four discarded RF excitations to allow for steady state equilibrium.

\section{Data Analysis}

Image preprocessing was performed using SPM99 (Wellcome Department of Cognitive Neurology, UK). Motion was detected by center of mass measurements. No volunteer had greater than a $3-\mathrm{mm}$ deviation in the center of mass in the $x$-, $y$-, or $z$-dimensions. Images were temporally adjusted for interleaved slice acquisition and realigned to the tenth image to correct for head movements between scans. The realigned scans were then normalized to the Montréal Neurologic Institute (MNI) Template found in SPM99 (www.fil.ion.ucl.ac.uk/ spm) by applying a 12-parameter affine transformation with nonlinear deformations routine (Friston et al., 1995). This template conforms to the MNI's standardized brain space and closely approximates Talairach and Tournoux's (1988) stereotaxic atlas. The functional data were high-pass-filtered and spatially smoothed with an 8-mm isotropic Gaussian kernel before statistical analysis. These normalized and smoothed data were used in the analysis procedures described below. 
Epochs synchronized to the onsets of the reaching-tograsp movements and containing two images preceding and nine images following events were extracted from the continuous time series of image volumes. Epochs were segregated and averaged by correct and incorrect trials. The average BOLD intensity signal values were converted to percent signal change relative to the 3 -sec prestimulus baseline. Voxel-based analyses identified activity associated with correct and incorrect reachingto-grasp movements through correlational analyses with a canonical reference waveform. We specified a reference waveform that increased after the onset of the reaching-to-grasp movement. On a subject-by-subject basis, the 18-sec raw signal change time courses from each voxel were correlated with the reference waveform and $t$ statistics were calculated for the correlation coefficients. This process, performed separately for the correct and incorrect conditions, provided two wholebrain normalized $t$ maps for each subject.

The individual subject $t$ maps were used as the basis of random-effects analysis across subjects. For each voxel in the Talairach space (Talairach \& Tournoux, 1988), each group of $t$ values (two derived from each subject) were tested for a significant difference from zero. The threshold for significance was set at a voxelwise uncorrected $p<.0001$ (two-tailed) and a spatial extent of three contiguous uninterpolated voxels for most analyses. The result was two whole-brain Talairach-normalized maps (one for correct and the other for incorrect movements) of significance values from the random-effects analysis. Lower significance values indicated a higher positive correlation between a voxel's waveform and the reference waveform.

For the final activation map, we were primarily interested in the intersection of the Talairach-normalized statistical maps for the correct and incorrect conditions. This intersection contained voxels that showed a significant (as defined above) positive response to both correct and incorrect trials (i.e., correct $\cap$ incorrect). We report activation locations from this map that reached a minimum cluster size of three contiguous uninterpolated voxels. We visualized these activations on the single subject T1 image available in SPM99. Aided by human brain atlases, we localized each cluster of activation and we report the anatomical label and MNI coordinates of the peak activation in Table 1. Eight (four anatomical locations by two hemispheres) ROI were identified. We then counted the number of activated uninterpolated voxels in each ROI by hemisphere. These counts were used to calculate the LI (LI = number of right hemisphere voxels / number of left hemisphere voxels) for each ROI. Increasing numbers in this index indicated greater right hemisphere laterality.

We conducted waveform analyses using the ROI identified as described above. The average signal from all voxels within each ROI was computed for each of the 12 time points and plotted to visualize the time course of the HDR during correct versus incorrect trials. Within each ROI averages of the percent change in signal intensity from baseline to $4.5-9 \mathrm{sec}$ after stimulus onset (four time points) were calculated for each condition as measures of waveform peak amplitude. A set of four 2 (hemisphere: right, left) $\times 2$ (condition: incorrect, correct) repeated measures ANOVAs was conducted, one analysis for each anatomical location, to evaluate differences in this amplitude measure as a function of stimulus condition. These analyses, which allowed us to test an a priori defined set of hypotheses concerning amplitude differences as a function of stimulus condition and ROI, constituted the primary analysis of the data.

\section{Acknowledgments}

This research was supported by the Department of Veterans Affairs (DVA) and NIMH grant MH-05286. Dr. Pelphrey was supported by NICHD grant 1-T32-HD40127 and NIH grant 1U54-MH66418-NIH. Dr. Morris was supported by NIH grant 1U54-MH66418-NIH. Dr. McCarthy was supported by a Career Research Scientist award from the DVA. We thank Ronald Viola, Julian Lim, Kari Karcher, Mary-Beth Nebel, Christopher Petty, Charles Michelich, and Allen Song for their assistance in stimulus development, data acquisition, data analysis, and/or manuscript preparation.

Reprint requests should be sent to Gregory McCarthy, DukeUNC Brain Imaging and Analysis Center, Duke University Medical Center, P.O. Box 3918, 163 Bell Building, Durham, NC 27710, or via e-mail: gregory.mccarthy@duke.edu.

The data reported in this experiment have been deposited in the fMRI Data Center (http://www.fmridc.org). The accession number is $2-2004-116 \mathrm{ND}$.

\section{Note}

1. For illustration of the STS-MTN5 complex localization, compare Figure 2 (panels 1-2 and 6-8) of this article to the lower right inset of Figure 4 of Wright et al. (2003), Figure 7 of Puce et al. (1998), and Figure 1 of McCarthy et al. (1995).

\section{REFERENCES}

Adolphs, R. (2003). Cognitive neuroscience of human social behaviour. Nature Reviews Neuroscience, 4, 65-78.

Allison, T., Puce, A., \& McCarthy, G. (2000). Social perception from visual cues: Role of the STS region. Trends in Cognitive Science, 4, 267-278.

Anscombe, G. E. M. (1957). Intention. Oxford: Basil Blackwell. Arbib, M. A., Billard, A., Iacoboni, M., \& Oztop, E. (2000). Synthetic brain imaging: Grasping, mirror neurons and imitation. Neural Networks, 13, 975-997.

Astington, J., \& Gopnik, A. (1991). Understanding desire and intention. In A. Whiten (Ed.), Natural theories of mind: The evolution, development and simulation of second-order representations (pp. 39-50). Oxford: Basil Blackwell.

Avikainen, S., Wohlschlager, A., Liuhanen, S., Hanninen, R., \& Hari, R. (2003). Impaired mirror-image imitation in asperger and high-functioning autistic subjects. Current Biology, 13, 339-341. 
Baron-Cohen, S. (1995). Mindblindness: An essay on autism and "theory of mind." Cambridge: MIT Press.

Beauchamp, M. S., Lee, K. E., Haxby, J. V., \& Martin, A. (2003). fMRI responses to video and point-light displays of moving humans and manipulable objects. Journal of Cognitive Neuroscience, 15, 991-1001.

Bekkering, H., Wohlschlager, A., \& Gattis, M. (2000). Imitation of gestures in children is goal-directed. Quarterly Journal of Experimental Psychology A, 53, 153-164.

Blakemore, S. J., Boyer, P., Pachot-Clouard, M., Meltzoff, A., Segebarth, C., \& Decety, J. (2003). The detection of contingency and animacy from simple animations in the human brain. Cerebral Cortex, 13, 837-844.

Bonda, E., Petrides, M., Ostry, D., \& Evans, A. (1996). Specific involvement of human parietal systems and the amygdala in the perception of biological motion. Journal of Neuroscience, 16, 3737-3744.

Brass, M., Bekkering, H., Wohlschlager, A., \& Prinz, W. (2000). Compatibility between observed and executed finger movements: Comparing symbolic, spatial, and imitative cues. Brain Cognition, 44, 124-143.

Brothers, L. (1990). The social brain: A project for integrating primate behavior and neurophysiology in a new domain. Concepts in Neuroscience, 1, 27-51.

Buccino, G., Binkofski, F., Fink, G. R., Fadiga, L., Fogassi, L., Gallese, V., Seitz, R. J., Zilles, K., Rizzolatti, G., \& Freund, H. J. (2001). Action observation activates premotor and parietal areas in a somatotopic manner: An fMRI study. European Journal of Neuroscience, 13, 400-404.

Culham, J. (2004). Human brain imaging reveals a parietal area specialized for grasping. In N. Kanwisher \& J. Duncan (Eds.), Attention and performance XX. Functional neuroimaging of visual cognition (pp. 415-436). Oxford: Oxford University Press.

Culham, J. C., Danckert, S. L., DeSouza, J. F., Gati, J. S., Menon, R. S., \& Goodale, M. A. (2003). Visually guided grasping produced fMRI activation in dorsal but not ventral stream brain areas. Experimental Brain Research, 153, 180-189.

Culham, J. C., \& Kanwisher, N. G. (2001). Neuroimaging of cognitive functions in human parietal cortex. Current Opinion in Neurobiology, 11, 157-163.

Decety, J., \& Grèzes, J. (1999). Neural mechanisms subserving the perception of human actions. Trends in Cognitive Science, 3, 172-178.

Dennett, D. C. (1987). The intentional stance. Cambridge: MIT Press.

Feldman, R., \& Reznick, J. S. (1996). Maternal perception of infant intentionality at 4 and 8 months. Infant Behavior and Development, 19, 483-496.

Gelman, R., \& Spelke, E. S. (1981). The development of thoughts about animate and inanimate objects: Implications for research in social cognition. In J. H. Flavell \& L. Ross (Eds.), The development of social cognition in children (pp. 43-66). Cambridge: Cambridge University Press.

Glover, G. H., \& Law, C. S. (2001). Spiral-in/out BOLD fMRI for increased SNR and reduced susceptibility artifacts. Magnetic Resonance Imaging in Medicine, 46, 515-522

Goschke, T., \& Kuhl, J. (1993). Representation of intentions: Persisting activation in memory. Journal of Experimental Psychology: Learning, Memory, and Cognition, 19, 1211-1226.

Grèzes, J., \& Decety, J. (2002). Does visual perception of object afford action? Evidence from a neuroimaging study. Neuropsychologia, 40, 212-222.

Grèzes, J., Fonlupt, P., Bertenthal, B., Delon-Martin, C., Segebarth, C., \& Decety, J. (2001). Does perception of biological motion rely on specific brain regions? Neuroimage, 13, 775-785.

Grossman, E. D., \& Blake, R. (2001). Brain activity evoked by inverted and imagined biological motion. Vision Research, 41, 1475-1482.

Grossman, E. D., \& Blake, R. (2002). Brain areas active during visual perception of biological motion. Neuron, 35 , $1167-1175$.

Guo, H., \& Song, A. W. (2003). Single-shot spiral image acquisition with embedded $z$-shimming for susceptibility signal recovery. Journal of Magnetic Resonance Imaging, 18, 389-395.

Heider, F., \& Simmel, M. (1944). An experimental study of apparent behavior. American Journal of Psychology, 57, $243-259$.

Iacoboni, M., Koski, L. M., Brass, M., Bekkering, H., Woods, R. P., Dubeau, M. C., Mazziotta, J. C., \& Rizzolatti, G. (2001). Reafferent copies of imitated actions in the right superior temporal cortex. Proceedings of the National Academy of Sciences, U.S.A., 98, 13995-13999.

Iacoboni, M., Woods, R. P., Brass, M., Bekkering, H., Mazziotta, J. C., \& Rizzolatti, G. (1999). Cortical mechanisms of human imitation. Science, 286, 2526-2528.

Karnath, H. O. (2001). New insights into the functions of the superior temporal cortex. Nature Reviews Neuroscience, 2, 568-576.

Klin, A. (2000). Attributing social meaning to ambiguous visual stimuli in higher-functioning autism and Asperger syndrome: The social attribution task. Journal of Child Psychology and Psychiatry, 41, 831-846.

Kunda, Z. (1999). Determining what is, what was, and what might have been: Hypothesis testing, covariation detection, and counterfactual thinking. In D. J. Ingle, M. A. Goodale, \& R. J. W. Mansfield (Eds.), Social cognition: Making sense of people (pp. 111-160). Cambridge: MIT Press.

McCarthy, G., Spicer, M., Adrignolo, A., Luby, M., Gore, J., \& Allison, T. (1995). Brain activation associated with visual motion studied by functional magnetic resonance imaging in humans. Human Brain Mapping, 2, 234-243.

Pelphrey, K. A., Mitchell, T. V., McKeown, M. J., Goldstein, J., Allison, T., \& McCarthy, G. (2003). Brain activity evoked by the perception of human walking: Controlling for meaningful coherent motion. Journal of Neuroscience, 23, 6819-6825.

Pelphrey, K. A., Singerman, J. D., Allison, T., \& McCarthy, G. (2003). Brain activation evoked by perception of gaze shifts: The influence of context. Neuropsychologia, 41, 156-170.

Pelphrey, K. A., Viola, R. J., \& McCarthy, G. (2004). When strangers pass: Processing of mutual and averted gaze in the superior temporal sulcus. Psychological Science, 15, 598-603.

Perrett, D. I., Harries, M. N., Bevan, R., Thomas, S., Benson, P. J., Mistlin, A. J., Chitty, A. J., Hietanen, J. K., \& Ortega, J. E. (1989). Frameworks of analysis for the neural representation of animate objects and action. Journal of Experimental Biology, 146, 87-114.

Perrett, D. I., Smith, P. A., Potter, D. D., Mistlin, A. J., Head, A. S., Milner, A. D., \& Jeeves, M. A. (1985). Visual cells in the temporal cortex sensitive to face view and gaze direction. Proceedings of the Royal Society of London, B: Biological Sciences, 22, 223, 293-317.

Perrett, D. I., Smith, P., Mistlin, A., Chitty, A., Head, A., Potter, D., Broennimann, R., Milner, A., \& Jeeves, M. (1985). Visual analysis of body movements by neurones in the temporal cortex of the macaque monkey: A preliminary report. Behavioural Brain Research, 16, 153-170. 
Puce, A., Allison, T., Bentin, S., Gore, J. C., \& McCarthy, G. (1998). Temporal cortex activation in humans viewing eye and mouth movements. Journal of Neuroscience, 18, 2188-2199.

Rizzolatti, G., \& Arbib, M. A. (1998). Language within our grasp. Trends in Neuroscience, 21, 188-194.

Talairach, J., \& Tournoux, P. (1988). Co-planar stereotaxic atlas of the buman brain. New York: Thieme.

Tootell, R. B., Reppas, J. B., Dale, A. M., Look, R. B., Sereno, M. I., Brady, T. J., \& Rosen, B. R. (1995). Visual motion aftereffect in human cortical area MT revealed by functional magnetic resonance imaging. Nature, 375, 139-141.

Vaina, L. M., Solomon, J., Chowdhury, S., Sinha, P., \& Belliveau, J. W. (2001). Functional neuroanatomy of biological motion perception in humans. Proceedings of the National Academy of Sciences, U.S.A., 98, 11656-11661.

Voyvodic, J. T. (1999). Real-time fMRI integrating paradigm control, physiology, behavior, and on-line statistical analysis. Neuroimage, 10, 91-106.

Wapner, S., \& Cirillo, L. (1968). Imitation of a model's hand movements: Age changes in transposition of left-right relations. Child Development, 39, 887-894.

Watson, J. D., Myers, R., Frackowiak, R. S., Hajnal, J. V., Woods, R. P., Mazziotta, J. C., Shipp, S., \& Zeki, S. (1993). Area V5 of the human brain: Evidence from a combined study using positron emission tomography and magnetic resonance imaging. Cerebral Cortex, 3, 79-94.

Wright, T. M., Pelphrey, K. A., Allison, T., McKeown, M. J., \& McCarthy, G. (2003). Polysensory interactions along lateral temporal regions evoked by audiovisual speech. Cerebral Cortex, 13, 1034-1043.

Zeki, S., Watson, J. D. G., Lueck, C. J., Friston, K. J., Kennard, C., \& Frackowiak, R. S. J. (1991). A direct discrimination of functional specialization in human visual cortex. Journal of Neuroscience, 11, 641-649. 